\title{
Razor-blade of life
}

SIR - Occam's razor is often justified on the grounds that our world is simple, as proved by fundamental physics. Equally often, Occam is declared wrong on the grounds that there is complexity in the world, for instance in biology. In both cases, Occam's razor is interpreted as a statement about the world, rather than a principle of scientific method. In his compelling discussion of Hume's problem (Nature 366, 105-106; 1993), Allan Goddard Lindh reopens this issue. But my view is that the interpretation of Occam's razor as a statement about the world is of no interest to the practising scientist. This argument does not involve any new philosophical idea.

A careful reading of Occam's statements "Plurality is not to be assumed without necessity" and "What can be done with fewer assumptions is done in vain with more", shows that Occam's principle is independent of the nature of the world: in any possible universe, we should seek the simplest feasible interpretation of our observations, even though this interpretation may be very complex in absolute terms.

As an example, a double helix is more complex that a single helix, but it is still the simplest structure that can account for what is known about DNA and heredity. No doubt an explanation involving a more complex structure could be contrived, but it is not necessary.

As Karl Popper pointed out in The Logic of Scientific Discovery, Occam's razor is a consequence of the requirement that hypotheses be falsifiable: if a theory contains fewer parameters than the facts that it can predict, then the theory has already been corroborated.

For instance, if ten points on a graph lie on a straight line, then the straight-line hypothesis could have been falsified eight times, but was not. In this case, a falsificationist application of Occam's razor requires that we accept the straight line as a working hypothesis, extrapolate from it, and test its predictions; it does not require accepting the straight line uncritically.

There are two other methodological reasons to seek simplicity: simple models are easier to simulate and they give more insight into the processes that one is trying to understand. However, I believe that the greater falsifiability of simpler hypotheses is the main reason why, in any possible world, we can do no better than trying out the simplest hypothesis first, independently of whether it is more likely to be true.

\section{Arthur Pece}

Department of Visual Science,

Institute of Ophthalmology,

Bath Street

London EC1V 9EL, UK
SIR - It is not surprising that Lindh concludes that Popper did not really succeed in solving Hume's problem of induction. Given that falsification is merely another (that is, negative) form of generalization of empirical observations, its methodological justification must undergo the same severe criticism as has already been formulated for its logical counterpart (that is, verification).

But it is surprising that Lindh totally neglects the conceptual starting point of all of Popper's well-known objections to inductive procedures. A closer look at the totality of Popper's work clearly reveals that his main concern was regularly concentrated on the categorical rejection of the commonly accepted idea that intelligent organisms are instructed about the environment by a simple assimilation of external information. This point is much more important than the purely methodological controversy. It leads directly to the fundamental problem of the origin of any kind of knowledge and, by doing so, goes far beyond the subordinate question about the functional relationships between direct perception and (always conjectural) thinking.

Modern physics, however, is probably not the scientific discipline to provide appropriate answers and to solve this basic problem. Rather, it is the task of biology to explain how living systems became able to transform 'senseless' physical information into (within the system) meaningful knowledge. At the time of Hume, it was not yet possible to imagine a phylogenetic evolution of human rationality itself, but today this is an idea strongly suggested by both the empirical and theoretical results of comparative ethology and evolutionary epistemology. Popper, by adding the term 'evolutionary' to his theory of Objective Knowledge (1972), did nothing more than to address exactly this issue.

\section{Adolf Hesch!}

Konrad Lorenz Institut,

A-3422 Altenberg/Donau,

Adolf-Lorenz-Gasse 2, Austria

\section{Don't call us ...}

SIR - Most journals now promise rapid review and publication, but, I wondered, has this recent emphasis on speed made any difference? Accordingly, I determined the time required for review and publication of papers that I submitted to peer review from 1977 to 1993.

Average reviewing time ( \pm s.d.) stayed constant at $8.7 \pm 2.3$ weeks in $1977-84$ ( $n$ $=9), 8.7 \pm 2.7$ weeks in $1985-89(n=10)$, and $9.2 \pm 3.0$ weeks in $1990-93(n=8)$. I defined reviewing time as the interval between the date the editor received the paper and the date on the letter enclosing the reviews. The fastest review, 4 weeks, was in 1987, and the slowest, 15 weeks, in 1988. Interestingly, much of this time was not used by reviewers. Papers commonly spent several weeks on an editor's desk both before being sent out for review and after the reviews were returned. (Editors' assistants seldom cover for their bosses.) On the other hand, the average time between acceptance and publication decreased from 18.7 weeks in 1977-84 to 14.2 weeks in 1990-93, ranging from 8 weeks, in 1978, to 35 weeks, in 1984 .

If my experience is general, then 8 or 9 weeks may represent a practical limit that balances the patience of authors with the workloads of editors and referees. Given this seemingly immutable barrier, I plan, though cannot promise, to leave editors in peace for a couple of months after submitting a paper. Should any journal actually complete a review within the promised 2-3 weeks, I shall, of course, be delighted. Jeffrey Boone Miller

Neuromuscular Laboratory,

Massachusetts General Hospital,

Charlestown, Massachusetts 02129, USA

\section{Food for worms}

SIR - As a formulation chemist, I am interested in a recent notion put forward by Daedalus (Nature 366, 20; 1993) regarding sustained release of pharmaceuticals by intestinal worms. By coupling the production of the pharmaceutical to some internal clock of the worms, pulsatile drug release could be obtained, which for some treatments is more advantageous than steady release. But the increased cost of feeding all these worms human cuisine would far outweigh the benefits obtained by steady dosing.

Actually, the best use of such worms would be in the control of human eating disorders, a fact that Daedalus all too quickly glosses over. Specifically developed tapeworms could be constructed for the over-indulgent and such disorders as anorexia nervosa and bulimia would disappear. Cleavage sites engineered into different sections of the worm could be actuated by various orally active enzymes that would tailor the worm's length and, therefore, its metabolism to provide an appropriate level of stasis for any individual. Any increased food consumption (no doubt some people will exploit the system with ever increasing times at the feed-bag) would be more than compensated with the millions saved by dispensing with fitness centres, exercise machines and expensive fly-by-night diet plans. It would appear that Daedalus missed the boat on this one.

David F. Johnson

557A South Dove Road,

Yardley, Pennsylvania 19067, USA 